\title{
ANÁLISIS DE LA PRODUCCIÓN Y DE LA VISIBILIDAD CIENTÍFICA DE ECUADOR EN EL CONTEXTO ANDINO (2000-2013)
}

\author{
Analysis of production and scientific visibility of \\ Ecuador in the Andean context (2000-2013)
}

Patricio Álvarez-Muñoz y Mario Pérez-Montoro

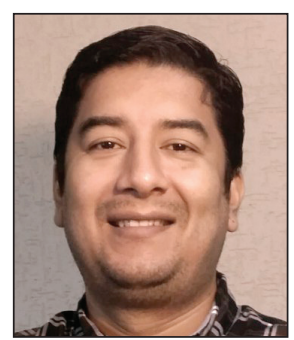

Patricio Álvarez-Muñoz es economista por la Escuela Superior Politécnica del Litoral en Ecuador y posgraduado en administración de empresas por la Universidad Tecnológica Empresarial de Guayaquil en Ecuador y ha realizado estancias de investigación en diversas universidades españolas. Es profesor de la Facultad de Ciencias Administrativas de la Universidad Estatal de Milagro en las materias de microeconomía y estadística. Cursa el Doctorado de Información y Documentación en la Sociedad del Conocimiento de la Universidad de Barcelona e investiga sobre modelos de negocio en la distribución de información científica.

http://orcid.org/0000-0002-9754-8050

Universidad Estatal de Milagro Ciudadela Universitaria, Km. 1.5 vía Milagro Km. 26. Milagro, Ecuador palvarezm@unemi.edu.ec

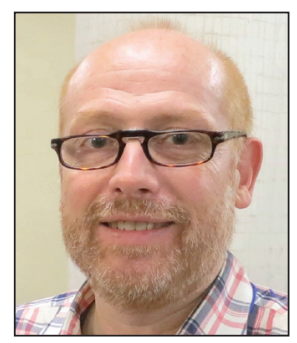

\begin{abstract}
Mario Pérez-Montoro es doctor en filosofía y ciencias de la educación por la Universidad de Barcelona y posgraduado en organización de sistemas de documentación por la Universidad Politécnica de Cataluña. Ha realizado estudios de posgrado en el Istituto di Discipline della Comunicazione de la Università di Bologna (Italia), y ha sido profesor visitante del CSLI (Center for the Study of Language and Information) de la Stanford University (California, EUA) y de la School of information de la UC Berkeley (California, EUA). Investiga sobre arquitectura y visualización de la información. Ha publicado los libros Arquitectura de la información en entornos web (Trea, 2010), The phenomenon of information (Scarecrow Press, 2007) y Gestión del conocimiento en las organizaciones (Trea, 2008), entre otros. Es profesor de la Facultad de Biblioteconomía y Documentación de la Universidad de Barcelona.
\end{abstract}

http://orcid.org/0000-0003-2426-8119

Universitat de Barcelona, Facultat de Biblioteconomia i Documentació Melcior de Palau, 140. 08034 Barcelona, España perez-montoro@ub.edu

\section{Resumen}

Se realiza un análisis cuantitativo de la producción científica del Ecuador en el contexto andino durante el período 20002013, tomando en consideración los datos suministrados por el SCImago journal \& country rank (SJR). A partir de esta información hemos identificado los indicadores de producción absoluta y de tasa de crecimiento; para la visibilidad, identificamos los indicadores de citación absoluta, citas por documentos y de colaboración internacional. Resultados: 1) se constata un crecimiento interanual positivo de la producción científica; 2) la mitad de la producción se adscribe a las áreas de agricultura y medicina, mostrándose como las principales áreas del desarrollo científico nacional; 3) el área más productiva, agricultura, también es la más citada.

\section{Palabras clave}

Producción científica; Bases de datos; Educación superior; Evaluación de la investigación; Colaboración internacional; SJR; Ecuador; Colombia; Perú; América Latina.

\section{Abstract}

A quantitative analysis of the scientific production of Ecuador in the Andean context is provided for 2000-2013, using data provided by the SCImago journal \& country rank $(S J R)$. We identified the indicators of absolute production and growth rate, and used three indicators to evaluate visibility: absolute citation, cites per document and international collaboration. We concluded that 1) Ecuador enjoyed positive growth in scientific production during the study period, except in 2010 
and $2011 ; 2$ ) it rebounded at the end of the period analyzed; 3) half of the production was in the areas of agriculture and medicine, which therefore appeared as the main areas of national scientific development; and 4) the most productive area, agriculture, was also the most cited.

\section{Keywords}

Scientific production; Databases; Higher education; Research assessment; International collaboration; SJR; Ecuador; Colombia; Perú; Latin America.

Álvarez-Muñoz, Patricio; Pérez-Montoro, Mario. (2015). “Análisis de la producción y visibilidad científica en Latinoamérica: el caso de Ecuador en el contexto andino (2000-2013)". El profesional de la información, v. 24, n. 5, pp. 577-586.

http://dx.doi.org/10.3145/epi.2015.sep.07

\section{Introducción}

Los procesos de generación y difusión de producción científica de calidad internacional deben ser considerados como mecanismos dinámicos y a la vez complejos, relacionados con la economía de los países y regiones. Ecuador es un país de economía pequeña y emergente, dependiente en gran parte del petróleo, que está realizando esfuerzos considerables para ubicarse en el mapa de países creadores de conocimiento de calidad. Latinoamérica, como generador de información científica, pasó de aportar el 2,3\% mundial en 2003 (Conicyt, 2014), al 4,03\% en 2013 (SJR, 2014). Ecuador se encuentra en la posición 12 en América Latina. En el período de análisis el país ha pasado de aportar al total latinoamericano el 0,39\% de producción científica en 2000 al 0,63\% en 2013 (SJR, 2014).

La evolución de la producción científica de las universidades ecuatorianas ha seguido un patrón similar al de otros centros de educación superior latinoamericanos. La falta de incentivos gubernamentales y el crecimiento desregularizado de centros privados en décadas anteriores dio lugar a un escenario de escasa producción científica. Sin embargo, medidas administrativas recientes de respaldo económico a la universidad pública parece que están cambiando esta situación. Para entender que los países latinoamericanos han tenido caminos difíciles de democratización de la educación superior hay que considerar los tres aspectos que han marcado históricamente la educación superior en Latinoamérica (Brunner; Ferrada-Hurtado, 2011; Levy, 2011; OECD, 2012):

- la mayoría de los estudiantes de pregrado optan por matricularse en instituciones de educación superior privadas;

- la mayoría de las instituciones de educación superior son privadas;

- una gran parte de los recursos invertidos en las instituciones de educación superior provienen del sector privado.

En los países latinoamericanos los gobiernos no han ejercido influencia directa sobre las universidades privadas. Ello les confiere desde el punto de vista de la autonomía universitaria una especie de autarquía, que ha prevalecido incluso en las universidades estatales provocando en conjunto una escasa producción científica en las décadas pasadas.

Por otra parte, América Latina y Asia son las regiones que crecen más mundialmente en generación de conocimiento. Esto es debido a que ambas tienen una locomotora que las empuja: China en Asia y Brasil en América Latina, con más del $50 \%$ de la producción de la región.

Dentro de América Latina algunos países como Colombia, Ecuador y Perú -que comparten algunas similitudes como idioma, producción petrolera, costumbres y economías complementarias-, han aumentado el gasto público en educación superior dando lugar a una mayor generación de conocimiento en las universidades.

Colombia invirtió en educación superior en 2014 un 0,96\% de su PIB, y Ecuador un 2,12\%, convirtiéndose en el país latinoamericano que más invierte en educación superior, superando a Brasil y a Chile, que invirtieron un 0,95\% (tabla 1 , figura 1).

\section{Objetivo y metodología}

No existen estudios detallados destacables que analicen la evolución y situación de la producción científica ecuatoriana. El objetivo principal de este trabajo es realizar un análisis cuantitativo de la producción científica de Ecuador en el período 2000-2013 dentro del contexto andino. Debido a la escasa producción publicada en revistas indexadas en WoS, este análisis se va a centrar en Scopus. Este trabajo pretende ser una herramienta de información para el análisis de la producción científica de Ecuador, cuyos resultados puedan contrastarse con los estudios de otros autores, y a su vez, un

Tabla 1. Evolución anual del gasto dedicado a I+D respecto al PIB

\begin{tabular}{|c|c|c|c|}
\hline & Colombia & Ecuador & Perú \\
\hline 2000 & 0,96 & $\mathrm{~s} / \mathrm{d}$ & 0,11 \\
\hline 2001 & 1,04 & 0,06 & 0,11 \\
\hline 2002 & 0,95 & 0,06 & 0,10 \\
\hline 2003 & 0,90 & 0,07 & 0,11 \\
\hline 2004 & 0,93 & $\mathrm{~s} / \mathrm{d}$ & 0,16 \\
\hline 2005 & 0,87 & $\mathrm{~s} / \mathrm{d}$ & $\mathrm{s} / \mathrm{d}$ \\
\hline 2006 & 0,88 & 0,72 & $\mathrm{~s} / \mathrm{d}$ \\
\hline 2007 & 0,86 & 0,82 & $\mathrm{~s} / \mathrm{d}$ \\
\hline 2008 & 0,87 & 0,81 & $\mathrm{~s} / \mathrm{d}$ \\
\hline 2009 & 0,95 & 1,62 & $\mathrm{~s} / \mathrm{d}$ \\
\hline 2010 & 1,10 & 1,80 & $\mathrm{~s} / \mathrm{d}$ \\
\hline 2011 & 1.01 & 1,59 & $\mathrm{~s} / \mathrm{d}$ \\
\hline 2012 & 0,96 & 1,55 & $\mathrm{~s} / \mathrm{d}$ \\
\hline 2013 & 0,96 & 1,83 & $\mathrm{~s} / \mathrm{d}$ \\
\hline 2014 & 0,96 & 2,12 & $\mathrm{~s} / \mathrm{d}$ \\
\hline
\end{tabular}

Datos suministrados por Ricyt 2015, Senescyt (2013) y Ministerio de Educación de Colombia (2013) 
instrumento de ayuda adicional en la toma de decisiones de autoridades universitarias y gestores de políticas públicas educativas ecuatorianas.

Se han utilizado los datos suministrados por el SCImago journal \& country rank (SJR) y se ha identificado una serie de indicadores cuantitativos y de visibilidad (Codina-Canet; Olmeda-Gómez; Perianes-Rodríguez, 2013). Scopus permite obtener un conjunto mayor de datos necesarios para este análisis frente a los productos de Thomson Reuters debido a su mayor cobertura de fuentes, temáticas y países (Bosman et al., 2006).

Los indicadores considerados son los siguientes:

Cuantitativos:

- producción absoluta: número de documentos de cualquier tipo en los que interviene al menos un autor adscrito a una institución ecuatoriana;

- tasa de crecimiento: aumento productivo de un año con respecto al anterior, diferencia porcentual del número de trabajos en relación con el período anterior.

De visibilidad:

- citación absoluta: impacto de las publicaciones medido a partir de número real de citas recibidas por un trabajo, permitiendo analizar la repercusión de los trabajos científicos en la comunidad científica;

- citas por documentos: promedio de citas recibidas por el total de la producción científica;

- colaboración internacional: porcentaje de participación de científicos extranjeros en la producción científica ecuatoriana.

A partir de estos indicadores, se han ido desarrollado los siguientes apartados. En el apartado 3 se presenta una comparativa de la producción científica ecuatoriana frente a los países de su entorno geográfico y su evolución respecto a su PIB. En el apartado 4 se revisa la producción científica ecuatoriana por áreas temáticas y su colaboración internacional. El trabajo concluye con el apartado 5, en el que se discuten los resultados obtenidos y se extraen algunas conclusiones a partir de la revisión de trabajos previos acerca del tema analizado, como Concytec, 2014; Conicyt, 2014; y Molina-Molina; De-Moya-Anegón, 2013.

\section{Producción científica de}

\section{Ecuador en el contexto andino y} latinoamericano

Hasta comienzos de la primera década de este siglo, Ecuador estaba inmerso en un contexto político en el que el sector público se debilitaba insistentemente (Correa, 2009). En la década de los 80 y 90, el país ingresó en una etapa privatizadora durante la que se liberalizó gran parte de la economía, incluida la educación en todos sus niveles. Durante ese período, entre 1980 y 2004 se crearan 14 nuevas universidades, llegando a un total de 72 instituciones de educación supe- rior, 33 de ellas privadas (45\% del total). No fue hasta el año 2006 cuando Ecuador comienza a desarrollar su Sistema de Educación Superior al mismo ritmo que sus vecinos andinos Colombia y Perú.

Este escenario universitario provocó la restricción del acceso a las personas de escasos recursos debido a un modelo de negocio de altos precios en las matrículas y tasas por servicios administrativos, además de una baja inversión en investigación. El resultado de esta situación condujo a una limitada (en cantidad y calidad) producción y difusión de los trabajos científicos.

La falta de acceso a otras fuentes de información constituye un impedimento importante para dinamizar y fortalecer la producción científica ecuatoriana

A partir de 2010, esta situación empieza a cambiar con la entrada en vigor de la Ley Orgánica de Educación Superior (LOES) de Ecuador, que persigue estimular el aumento de la investigación científica de calidad e impacto en las universidades del país. Entre las propuestas adoptadas desde la LOES está la de impulsar mecanismos públicos que faciliten el acceso a las fuentes de información científica, insumos indispensables para la generación de conocimiento actualizados y de calidad.

En la actualidad la producción de información científica de Ecuador, como país de desarrollo tardío, es muy reducida, sobre todo si la comparamos con la de países latinoamericanos del entorno y de similares características socioeconómicas como Colombia y Perú. Según el $S J R$, Ecuador se encuentra en el puesto número 12 en América Latina. En el período 1996-2013 tuvo una producción de 5.365 artículos científicos, unos resultados muy modestos comparados con los 10.584 artículos científicos de Perú y los 43.554 de Colombia.

Las universidades públicas ecuatorianas tienen acceso a algunas bases de datos como Springer, Taylor \& Francis,

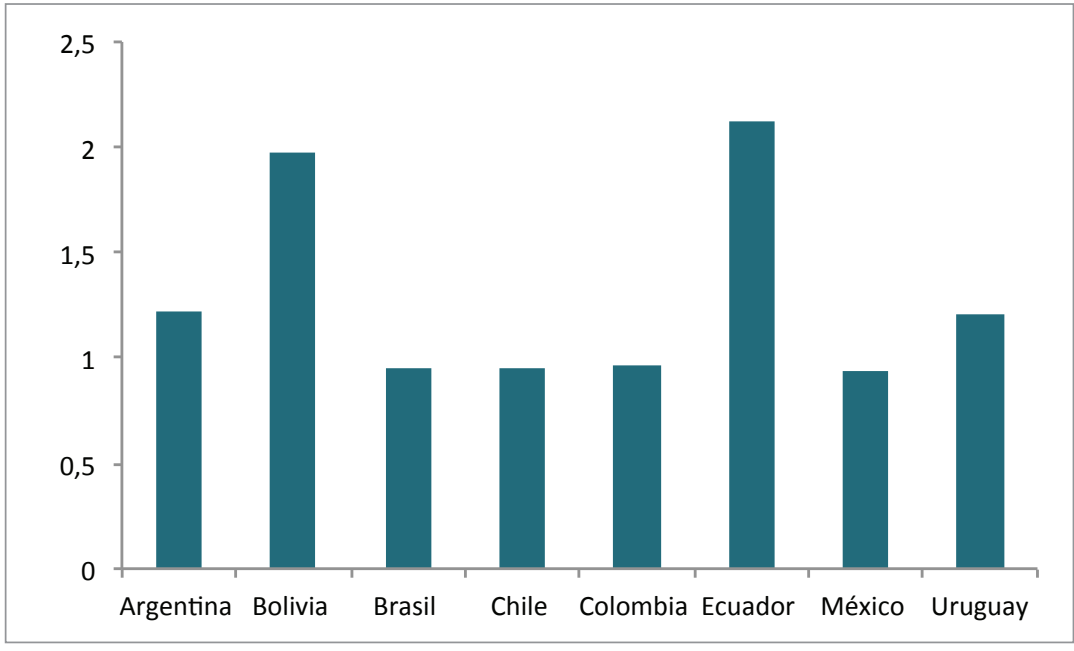

Figura 1. Inversión en educación superior en Latinoamérica en \% del PIB nacional Datos suministrados en Secretaría Nacional de Planificación y Desarrollo (2015) 


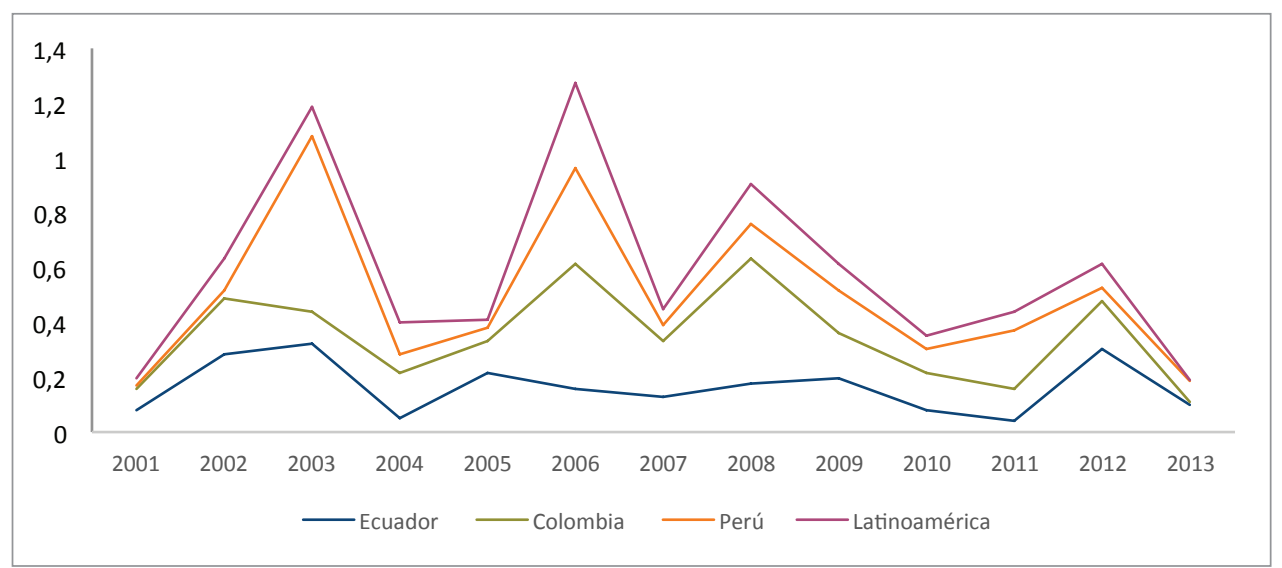

Figura 2. Evolución de la tasa de crecimiento de la producción comparada 2000-2013. Datos de SJR (2014) citable, citación y tasa de crecimiento de Ecuador, Colombia, Perú y América Latina en el período 20002013.

En la producción científica citable de Ecuador los años con mayor aportación al total regional fueron 2012 $(0,53 \%)$ y 2013 (0,58\%). Entre 2000 y 2011 su aportación se sitúa en el 0,47\%, muy baja con respecto a la de sus vecinos.

En citas, Ecuador representa menos del $1 \%(0,74 \%)$ de

Wiley, Gale o ProQuest. El acceso se realiza mediante el copago del $50 \%$ por parte del gobierno y el $50 \%$ por parte de la universidad. Sin embargo, estas bases de datos suponen sólo una parte de la información necesaria para el desarrollo de la actividad científica. La falta de acceso a otras fuentes constituye a día de hoy uno de los impedimentos más importantes para dinamizar y fortalecer la producción científica ecuatoriana. Se necesitaría un incremento de los recursos destinados a la educación superior. En esta línea, desde el gobierno ecuatoriano se están poniendo los medios para avanzar en la transformación de una matriz productiva basada en recursos finitos a otra basada en recursos intangibles como el conocimiento.

En este contexto, las publicaciones citables de Ecuador en el período $2000-2013$ ascienden a 4.563 y representan el $0,50 \%$ de la producción científica citable de América Latina. Los documentos de Ecuador, Colombia y Perú a su vez representan el 5,83\%. La tabla 2 muestra información detallada de los datos interanuales globales de producción científica la visibilidad total de América Latina. 2008, 2010 y 2012 son los años con mayor aportación, en torno al 1,07\%. Colombia con un 3,54\% y Perú con 1,49\%, dejan muy detrás a Ecuador. La visibilidad de la producción científica de Ecuador con respecto a América Latina es por tanto inferior a la de Colombia y Perú.

\section{El perfil científico latinoamericano tiene por eje dos áreas: medicina y agricultu- ra, y Ecuador no escapa de esa tendencia}

La figura 2 presenta la evolución del crecimiento de la producción. Se observa una evolución diferente a la de sus vecinos geográficos de referencia, caracterizada por un marcado crecimiento dispar de aumentos y reducciones que concluye con un final de ciclo con un leve crecimiento. Como contraste América Latina presenta un descenso continuo desde 2008.

Tabla 2. Producción científica y tasa de crecimiento comparada 2000-2013

\begin{tabular}{|c|c|c|c|c|c|c|c|c|c|c|c|c|}
\hline & \multicolumn{3}{|c|}{ Ecuador } & \multicolumn{3}{|c|}{ Colombia } & \multicolumn{3}{|c|}{ Perú } & \multicolumn{3}{|c|}{ América Latina } \\
\hline & Pc & $\mathbf{C i}$ & TcEcu & Pc & $\mathbf{C i}$ & TcCol & Pc & $\mathbf{C i}$ & TcPeru & Pc & $\mathbf{C i}$ & TCAL \\
\hline 2000 & 123 & 3.044 & & 808 & 14.407 & & 234 & 6.116 & & 31.451 & 537.430 & \\
\hline 2001 & 112 & 3.103 & $-0,09$ & 738 & 11.519 & $-0,09$ & 231 & 5.176 & $-0,01$ & 32.604 & 523.674 & 0,04 \\
\hline 2002 & 144 & 2.927 & 0,29 & 894 & 14.287 & 0,21 & 238 & 4.820 & 0,03 & 36.349 & 579.481 & 0,11 \\
\hline 2003 & 191 & 3.592 & 0,33 & 1.010 & 16.674 & 0,13 & 392 & 10.000 & 0,65 & 40.672 & 654.649 & 0,12 \\
\hline 2004 & 201 & 5.157 & 0,05 & 1.186 & 17.255 & 0,17 & 417 & 9.751 & 0,06 & 45.919 & 690.979 & 0,13 \\
\hline 2005 & 247 & 4.825 & 0,23 & 1.322 & 19.962 & 0,11 & 441 & 8.322 & 0,06 & 47.587 & 642.852 & 0,04 \\
\hline 2006 & 287 & 5.427 & 0,16 & 1.922 & 24.590 & 0,45 & 597 & 10.311 & 0,35 & 62.360 & 705.729 & 0,31 \\
\hline 2007 & 326 & 4.318 & 0,14 & 2.324 & 24.651 & 0,21 & 637 & 11.689 & 0,07 & 66.206 & 679.557 & 0,06 \\
\hline 2008 & 385 & 6.979 & 0,18 & 3.385 & 28.413 & 0,46 & 722 & 10.714 & 0,13 & 75.799 & 665.335 & 0,14 \\
\hline 2009 & 463 & 5.076 & 0,20 & 3.943 & 25.411 & 0,16 & 838 & 9.299 & 0,16 & 83.217 & 579.556 & 0,10 \\
\hline 2010 & 424 & 4.457 & 0,08 & 4.528 & 23.446 & 0,15 & 909 & 10.345 & 0,08 & 88.048 & 445.397 & 0,06 \\
\hline 2011 & 444 & 2.579 & 0,05 & 5.094 & 18.455 & 0,13 & 1.104 & 7.019 & 0,21 & 94.865 & 318.145 & 0,08 \\
\hline 2012 & 578 & 1.880 & 0,30 & 6.040 & 14.158 & 0,19 & 1.162 & 3.590 & 0,05 & 102.632 & 173.392 & 0,08 \\
\hline 2013 & 638 & 406 & 0,10 & 6.142 & 2.461 & 0,02 & 1.257 & 703 & 0,08 & 102.195 & 36.199 & 0,00 \\
\hline Total & 4.563 & 53.770 & & 39.336 & 255.689 & & 9.179 & 107.855 & & 909.904 & 7.232 .375 & \\
\hline
\end{tabular}

Pc: Producción citable (incluye artículos, resúmenes y comunicaciones a congresos); Ci: Citas; Tc: Tasa de crecimiento. Datos de SJR (2014) 
Tabla 3. Producción científica según Scopus en relación con el PIB período 2000-2011: publicaciones/PIB en miles de millones USD

\begin{tabular}{|c|c|c|c|c|c|c|c|c|c|c|c|c|}
\hline & $\mathbf{2 0 0 0}$ & $\mathbf{2 0 0 1}$ & $\mathbf{2 0 0 2}$ & $\mathbf{2 0 0 3}$ & $\mathbf{2 0 0 4}$ & $\mathbf{2 0 0 5}$ & $\mathbf{2 0 0 6}$ & $\mathbf{2 0 0 7}$ & $\mathbf{2 0 0 8}$ & $\mathbf{2 0 0 9}$ & $\mathbf{2 0 1 0}$ & $\mathbf{2 0 1 1}$ \\
\hline Ecuador & 8,54 & 5,57 & 7,24 & 7,10 & 5,25 & 6,41 & 5,46 & 6,50 & 6,23 & 7,84 & 6,04 & 5,46 \\
\hline Colombia & 7,35 & 7,47 & 8,33 & 8,87 & 7,77 & 8,48 & 6,85 & 5,97 & 9,06 & 10,20 & 9,48 & 9,50 \\
\hline Perú & 4,28 & 5,14 & 6,07 & 6,88 & 4,75 & 5,13 & 4,90 & 5,53 & 5,30 & 5,99 & 4,98 & 4,45 \\
\hline
\end{tabular}

Datos de la Red de Indicadores de Ciencia y Tecnología (Ricyt, 2014) y Bruque-Gámez (2014).

El incremento de las publicaciones de Ecuador en el período 2011-2012 ascendió a 134 documentos, representando un 0,56\% del crecimiento de la producción regional, una cifra que, a pesar del aumento, sigue siendo baja. En América Latina el mejor período es 2005-2006 con 14.773 documentos, mientras que en 2009-2011 hay un decrecimiento de la producción, repuntando en 2012 para volver a descender en 2013. A pesar del incremento en el gasto público en I+D, no se tradujo en un aumento de la tasa de crecimiento de la producción, debido a una posible falta de coordinación entre lo asignado a las instituciones de educación superior y la capacidad de generación de proyectos de investigación que desemboquen en artículos de carácter científico por parte de las universidades públicas.

Por otro lado, se observa también que la cantidad de publicaciones científicas aumenta junto al producto interior bruto (PIB, suma de la producción total de bienes y servicios de un país en un año). Este aumento paralelo se puede justificar por el supuesto de que un mayor PIB se traduce en mayor inversión en herramientas para investigación y producción intelectual.

En la tabla 3, con datos hasta 2011, se observa que Ecuador ha mantenido un comportamiento irregular con relación a sus países vecinos. Colombia se diferencia del resto gracias a las políticas de fomento al conocimiento y estímulo a la investigación desplegadas.

\section{Producción científica por áreas temáticas}

La producción desagregada por áreas temáticas no sólo refleja el grado de especialización sino también su capacidad para generar conocimiento y caracterizar la especialidad temática de las instituciones de un país.

La distribución temática porcentual de la producción de la figura 3 compara el patrón de publicación en Ecuador frente al de Colombia y Perú, comprobándose que existen diferencias entre esos patrones temáticos.

Ecuador publicó 238 documentos en agricultura, el área más productiva, que representa el $21,79 \%$ de la producción nacional en 2013. Con un valor similar a los de ingeniería se encuentra la medicina con el $20,6 \%$. A continuación, la producción de documentos en bioquímica, genética y biología molecular se sitúa en un $8,52 \%$, seguido por ciencias medioambientales con un $8,24 \%$ y ciencias sociales con un $5,68 \%$, por encima de ingeniería con un $3,39 \%$.

\section{Se evidencia un área emergente dentro del mapa de especialización de la ciencia ecuatoriana: las ciencias ambientales}

De acuerdo con el patrón denominado triángulo de la ciencia, las publicaciones no pertenecientes al área de ciencias sociales y humanidades, se distribuyen en tres ejes temáticos (Herrero-Solana; Vargas-Quesada, 2010):

- el grupo de mayor tamaño y cúspide del triángulo, corresponde con el dominio de las ciencias biomédicas: medicina, bioquímica, genética, biología molecular;

- el segundo grupo, de tamaño intermedio y zona media del triángulo, con las ciencias fundamentales (física, química, matemáticas) y las ingenierías;

- el tercer grupo, de menor tamaño y base del triángulo, con la agricultura.

Los resultados de Ecuador no concuerdan con la distribución de ese triángulo de la ciencia, ya que claramente se evidencia que el área de agricultura es la que presenta mayor producción, mientras que países como Colombia y Perú, sí que encajan en la distribución propuesta por el triángulo.

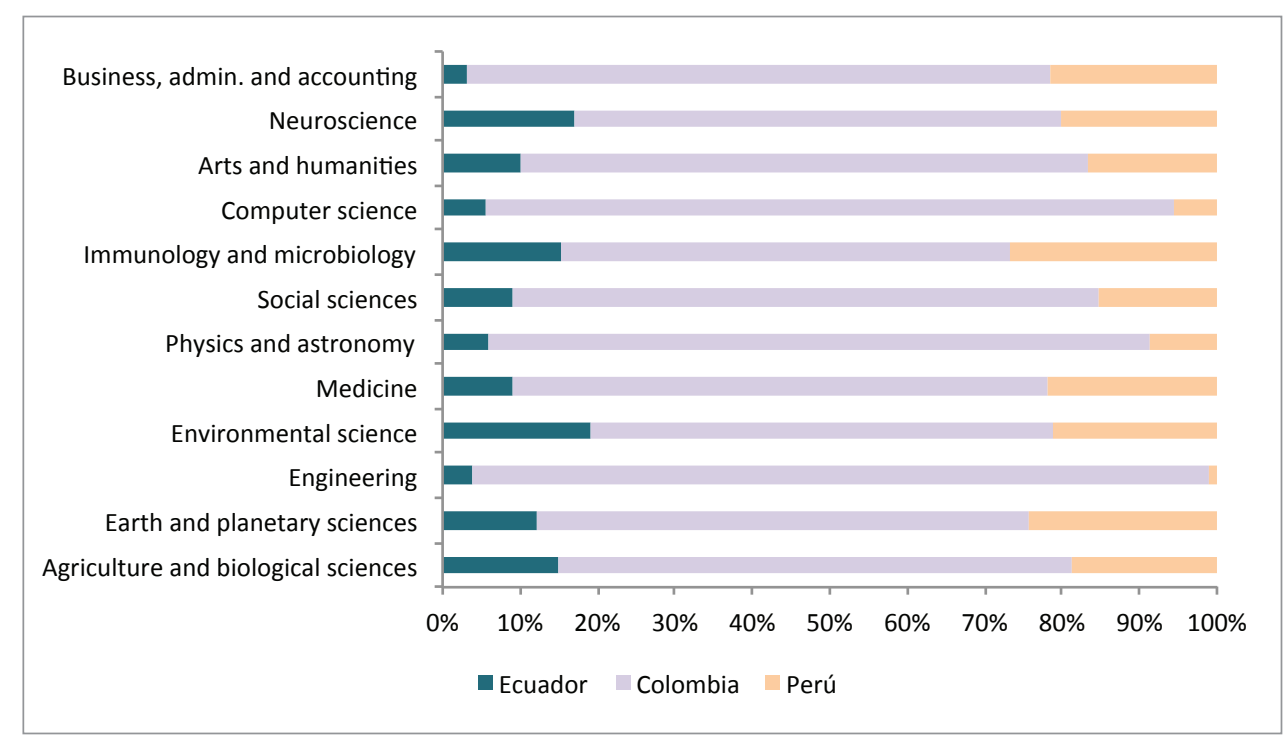

Figura 3. Distribución porcentual de la producción por áreas temáticas. Datos de SJR (2014) 
Tabla 4. Producción científica. Tasas de crecimiento y citas por documento de las áreas temáticas 2000-2013

\begin{tabular}{|c|c|c|c|c|c|c|c|c|c|c|c|c|c|c|c|c|}
\hline & $00-01$ & $01-02$ & $02-03$ & 03-04 & 04-05 & 05-06 & $06-07$ & $07-08$ & 08-09 & 09-10 & $10-11$ & $11-12$ & $12-13$ & CpD & TC & $\% \mathrm{Ci}$ \\
\hline $\begin{array}{l}\text { Agricultura y cien- } \\
\text { cias biológicas }\end{array}$ & 0,22 & $-0,54$ & $-0,47$ & $-0,10$ & $-0,04$ & $-0,25$ & $-0,32$ & 0,03 & $-0,46$ & 0,18 & $-0,14$ & $-0,28$ & $-0,17$ & 0,66 & 5,34 & 27,33 \\
\hline $\begin{array}{l}\text { Tierra y ciencias } \\
\text { planetarias }\end{array}$ & 0,10 & $-0,89$ & $-0,65$ & 0,32 & $-0,32$ & $-0,32$ & 0,03 & $-0,69$ & 0,28 & 0,15 & 0,12 & $-0,38$ & $-0,03$ & 0,80 & 3,10 & 8,89 \\
\hline Ingeniería & 0,14 & $-1,00$ & 0,50 & $-1,17$ & $-0,46$ & 0,11 & 0,12 & $-0,13$ & $-0,53$ & $-0,15$ & 0,10 & $-0,78$ & 0,23 & 0,30 & 4,14 & 2,30 \\
\hline $\begin{array}{l}\text { Ciencias ambien- } \\
\text { tales }\end{array}$ & $-0,89$ & $-0,41$ & $-0,58$ & $-0,11$ & 0,31 & 0,21 & $-0,91$ & 0,09 & $-0,55$ & $-0,21$ & 0,16 & $-0,41$ & $-0,01$ & 0,84 & 8,67 & 14,45 \\
\hline Medicina & 0,19 & $-0,11$ & $-0,82$ & 0,19 & $-0,08$ & $-0,21$ & 0,03 & $-0,20$ & $-0,25$ & $-0,07$ & 0,06 & $-0,22$ & $-0,35$ & 0,58 & 2,96 & 22,09 \\
\hline Física y astronomía & $-0,20$ & 0,04 & 0,48 & $-0,25$ & $-1,60$ & $-0,03$ & $-0,10$ & $-0,27$ & 0,00 & 0,20 & $-0,18$ & $-0,17$ & 0,16 & 0,78 & 1,60 & 11,52 \\
\hline Ciencias sociales & $-1,33$ & $-0,14$ & 0,25 & $-0,83$ & & 0,09 & 0,20 & $-0,75$ & $-1,07$ & 0,00 & $-0,14$ & $-0,06$ & $-0,77$ & 0,41 & 18,67 & 2,91 \\
\hline $\begin{array}{l}\text { Inmunología y } \\
\text { microbiología }\end{array}$ & 0,80 & $-2,50$ & $-2,00$ & 0,43 & $-0,17$ & 0,07 & 0,15 & $-1,00$ & $-0,50$ & 0,15 & 0,21 & $-0,64$ & $-0,47$ & 0,87 & 4,20 & 6,50 \\
\hline $\begin{array}{l}\text { Ciencias de com- } \\
\text { putación }\end{array}$ & 0,50 & $-6,00$ & 0,86 & 0,00 & $-3,00$ & $-2,25$ & $-0,15$ & 0,07 & $-0,57$ & $-0,14$ & 0,08 & $-0,83$ & 0,29 & 0,37 & 13,00 & 2,00 \\
\hline $\begin{array}{l}\text { Artes y humani- } \\
\text { dades }\end{array}$ & $-0,33$ & 0,50 & & $-2,50$ & 0,14 & & 0,50 & $-2,00$ & 0,22 & 0,29 & $-1,00$ & $-1,00$ & $-0,25$ & 0,10 & 7,00 & 0,27 \\
\hline Neurociencias & 0,00 & 0,29 & $-2,40$ & 0,59 & $-1,57$ & 0,17 & $-0,07$ & 0,50 & $-1,75$ & 0,45 & $-0,33$ & 0,06 & $-0,27$ & 0,27 & 1,67 & 1,18 \\
\hline $\begin{array}{l}\text { Negocios, admin. y } \\
\text { contabilidad }\end{array}$ & & & 1,00 & & & & $-2,00$ & 0,67 & $-7,00$ & 0,13 & $-0,43$ & 0,30 & 0,29 & 0,48 & & 0,55 \\
\hline
\end{tabular}

CpD: Citas por documento (total de citas del área/total de la producción); Ci: citación; \%Ci: citas del área/total de citas; TC: tasa de crecimiento. Datos de $S J R, 2014$

La tabla 4 muestra los resultados de la tasa de crecimiento y el promedio de citas por documento de cada área en Ecuador. El número medio de citas por documento es de 0,59. Los valores superiores al promedio más destacados son de física y astronomía con 1,87 y siguen tierra y ciencias planetarias y ciencias ambientales, ambas con 0,90 citas por documento.

La tabla 5 muestra un resumen de la anterior, ordenada por la producción de las áreas temáticas. Destacan agricultura y medicina, cuya producción conjunta representa el 50\% del total.

Tabla 5. Producción, citas y visibilidad por áreas temáticas de Ecuador (2000-2013)

\begin{tabular}{|c|l|c|c|c|c|c|}
\hline Puesto & \multicolumn{1}{|c|}{ Área temática } & Producción & $\%$ & CpD & TC & \%Ci \\
\hline 1 & Agricultura y ciencias biológicas & 1.503 & 26,0 & 0,66 & 5,34 & 27,3 \\
\hline 2 & Medicina & 1.388 & 24,0 & 0,58 & 2,96 & 22,1 \\
\hline 3 & Ciencias ambientales & 629 & 10,9 & 0,84 & 8,67 & 14,5 \\
\hline 4 & Física y astronomía & 539 & 9,3 & 0,78 & 1,60 & 12,0 \\
\hline 5 & Tierra y ciencias planetarias & 405 & 7,0 & 0,80 & 3,10 & 8,9 \\
\hline 6 & Ingeniería & 278 & 4,8 & 0,30 & 4,14 & 2,3 \\
\hline 7 & Inmunología y microbiología & 273 & 4,7 & 0,87 & 4,20 & 6,5 \\
\hline 8 & Ciencias sociales & 261 & 4,5 & 0,41 & 18,67 & 2,9 \\
\hline 9 & Ciencias de computación & 197 & 3,4 & 0,37 & 13,00 & 2,0 \\
\hline 10 & Neurociencias & 162 & 2,8 & 0,27 & 1,67 & 1,2 \\
\hline 11 & Arte y humanidades & 102 & 1,8 & 0,10 & 7,00 & 0,3 \\
\hline 12 & Negocios, admin. y contabilidad & 42 & 0,7 & 0,48 & 0 & 0,6 \\
\hline & Total & 5.779 & 100 & & & 100 \\
\hline
\end{tabular}

CpD: Citas por documento (total de citas del área/total de la producción); \%Ci: citas del área/total de citas; TC: tasa de crecimiento. Datos de SJR, 2014
A partir del año 2007 el área de agricultura es la que más desarrollo ha experimentado con un crecimiento a partir de 2000 del $534 \%$ de publicaciones científicas. A su vez, medicina en 2000 produjo 53 publicaciones llegando en 2013 a 210 , un incremento del $296 \%$ en dicho período. El perfil científico latinoamericano tiene por eje estas dos áreas: medicina y agricultura. Ecuador no escapa de esa tendencia, pero se evidencia un área emergente dentro del mapa de especialización de la ciencia ecuatoriana: las ciencias ambientales, que con una producción en el año 2000 de 9 publicaciones termina 2013 con 87 publicaciones científicas, lo que supone un incremento del $866 \%$, siendo un área de potencial consolidación debido a la situación geográfica de Ecuador.

La tabla 7 muestra la producción científica por áreas temáticas de Colombia. En este caso se observa que el perfil científico es similar al de Ecuador, teniendo como base las áreas de medicina y agricultura como las más punteras, aunque otras como ingeniería y física tienen un papel destacado. Cabe resaltar que la producción científica de Colombia es 8 veces superior a la de Ecuador y 4 veces la Perú, lo que la coloca como el país andino más dinámico en generación de conocimiento.

La tabla 8 muestra información de la producción científica por áreas temáticas de Perú. Como en los dos casos anteriores, medicina y agricultura son 
Tabla 6. Producción científica por áreas temáticas de Ecuador (2000-2013)

\begin{tabular}{|l|r|r|r|r|r|r|r|r|r|r|r|r|r|r|}
\hline & $\mathbf{2 0 0 0}$ & $\mathbf{2 0 0 1}$ & $\mathbf{2 0 0 2}$ & $\mathbf{2 0 0 3}$ & $\mathbf{2 0 0 4}$ & $\mathbf{2 0 0 5}$ & $\mathbf{2 0 0 6}$ & $\mathbf{2 0 0 7}$ & $\mathbf{2 0 0 8}$ & $\mathbf{2 0 0 9}$ & $\mathbf{2 0 1 0}$ & $\mathbf{2 0 1 1}$ & $\mathbf{2 0 1 2}$ & $\mathbf{2 0 1 3}$ \\
\hline Agricultura y ciencias biológicas & 35 & 26 & 43 & 62 & 69 & 72 & 89 & 119 & 113 & 166 & 136 & 155 & 196 & 222 \\
\hline Tierra y ciencias planetarias & 10 & 9 & 17 & 27 & 18 & 25 & 33 & 32 & 53 & 39 & 33 & 29 & 39 & 41 \\
\hline Ingeniería & 7 & 6 & 12 & 6 & 13 & 19 & 17 & 15 & 17 & 26 & 30 & 27 & 47 & 36 \\
\hline Ciencias ambientales & 9 & 17 & 24 & 37 & 42 & 29 & 23 & 43 & 39 & 61 & 73 & 62 & 83 & 87 \\
\hline Medicina & 53 & 40 & 45 & 80 & 66 & 69 & 86 & 87 & 103 & 128 & 141 & 127 & 153 & 210 \\
\hline Física y astronomía & 20 & 24 & 23 & 12 & 15 & 39 & 40 & 44 & 55 & 55 & 45 & 53 & 62 & 52 \\
\hline Ciencias sociales & 3 & 7 & 8 & 6 & 11 & 11 & 10 & 8 & 13 & 29 & 28 & 33 & 35 & 59 \\
\hline Inmunología y microbiología & 10 & 2 & 7 & 21 & 12 & 14 & 11 & 10 & 21 & 33 & 26 & 22 & 32 & 52 \\
\hline Ciencias de computación & 2 & 1 & 7 & 1 & 1 & 4 & 13 & 15 & 14 & 22 & 25 & 22 & 42 & 28 \\
\hline Artes y humanidades & 3 & 4 & 2 & 2 & 7 & 5 & 4 & 3 & 9 & 7 & 5 & 8 & 19 & 24 \\
\hline Neurociencias & 6 & 6 & 5 & 14 & 7 & 16 & 13 & 15 & 7 & 20 & 11 & 13 & 13 & 16 \\
\hline Negocios, admin. y contabilidad & 0 & 0 & 0 & 1 & 1 & 1 & 1 & 2 & 1 & 7 & 7 & 9 & 7 & 5 \\
\hline Totales & 15 & 142 & 193 & 269 & 262 & 304 & 340 & 393 & 445 & 593 & 560 & 560 & 728 & 832 \\
\hline
\end{tabular}

Datos de SJR, 2015

las áreas con mayor producción científica y delinean el perfil de la ciencia en ese país. Cabe señalar que las demás áreas tienen una producción muy similar pero nada significativa.

\section{Citas por áreas temáticas}

En la tabla 9 se puede observar que en 2013 las áreas que definen los perfiles científicos de los 3 países tienen diferentes grados de citación. En las áreas de medicina y agricultura, las más productivas en los tres países, Ecuador tiene un mejor nivel de citación.

\section{Colaboración internacional}

A pesar de ser el país con menor producción científica, Ecuador es el que cuenta con un mayor porcentaje de colaboración internacional a lo largo del período estudiado. El promedio para Ecuador es del 75,98\%, el de Colombia 52,37\% y de Perú $73,91 \%$. Esto coincide con el porcentaje de citas re- cibidas por las áreas temáticas, debido a que Ecuador por su alto porcentaje de colaboración internacional tiene trabajos de mayor calidad y esto influye directamente en el grado de citación de sus trabajos.

La colaboración internacional puede ser considerada como un síntoma de calidad, que tiene que ver con un proceso llamado de transferencia reputacional, ya que a medida que se colabora con importantes instituciones o centros de investigación de alto prestigio científico en el mundo, las posibilidades de hacer crecer el nivel medio de las investigaciones son mayores.

\section{Discusión y conclusiones}

\section{Crecimiento de la producción científica}

En Ecuador se constata un crecimiento interanual positivo de la producción científica excepto en los años 2010 y 2011,

Tabla 7. Producción científica por áreas temáticas de Colombia (2000-2013)

\begin{tabular}{|l|r|r|r|r|r|r|r|r|r|r|r|r|r|r|}
\hline & $\mathbf{2 0 0 0}$ & $\mathbf{2 0 0 1}$ & $\mathbf{2 0 0 2}$ & $\mathbf{2 0 0 3}$ & $\mathbf{2 0 0 4}$ & $\mathbf{2 0 0 5}$ & $\mathbf{2 0 0 6}$ & $\mathbf{2 0 0 7}$ & $\mathbf{2 0 0 8}$ & $\mathbf{2 0 0 9}$ & $\mathbf{2 0 1 0}$ & $\mathbf{2 0 1 1}$ & $\mathbf{2 0 1 2}$ & $\mathbf{2 0 1 3}$ \\
\hline Agricultura y ciencias biológicas & 123 & 138 & 159 & 181 & 233 & 232 & 339 & 350 & 567 & 707 & 789 & 881 & 1.098 & 992 \\
\hline Tierra y ciencias planetarias & 34 & 35 & 42 & 39 & 38 & 50 & 94 & 83 & 93 & 146 & 166 & 153 & 252 & 216 \\
\hline Ingeniería & 75 & 84 & 84 & 117 & 291 & 190 & 231 & 300 & 578 & 733 & 808 & 955 & 1040 & 894 \\
\hline Ciencias ambientales & 44 & 50 & 57 & 49 & 61 & 61 & 80 & 120 & 118 & 179 & 207 & 234 & 255 & 272 \\
\hline Medicina & 235 & 229 & 277 & 279 & 319 & 403 & 607 & 725 & 1.053 & 1.104 & 1.155 & 1.304 & 1.458 & 1.609 \\
\hline Física y astronomía & 198 & 141 & 135 & 197 & 237 & 212 & 293 & 301 & 450 & 383 & 445 & 593 & 748 & 771 \\
\hline Ciencias sociales & 30 & 26 & 26 & 44 & 35 & 47 & 53 & 106 & 147 & 272 & 310 & 374 & 451 & 484 \\
\hline Inmunología y microbiología & 59 & 37 & 59 & 62 & 72 & 82 & 137 & 117 & 129 & 163 & 175 & 223 & 217 & 196 \\
\hline Ciencias de computación & 29 & 27 & 28 & 79 & 104 & 85 & 144 & 205 & 253 & 228 & 435 & 580 & 700 & 459 \\
\hline Artes y humanidades & 13 & 15 & 8 & 13 & 21 & 25 & 26 & 61 & 81 & 125 & 138 & 164 & 185 & 177 \\
\hline Neurociencias & 14 & 9 & 15 & 21 & 27 & 23 & 20 & 35 & 31 & 42 & 46 & 57 & 59 & 59 \\
\hline Negocios, admin. y contabilidad & 4 & 7 & 7 & 11 & 7 & 15 & 23 & 38 & 71 & 90 & 86 & 107 & 132 & 116 \\
\hline Totales & 858 & 798 & 897 & 1.092 & 1.445 & 1.425 & 2.047 & 2.441 & 3.571 & 4.172 & 4.760 & 5.625 & 6.595 & 6.245 \\
\hline
\end{tabular}

Elaborado a partir de los datos de SJR, 2015 
Tabla 8. Producción científica por áreas temáticas de Perú (2000-2013)

\begin{tabular}{|c|c|c|c|c|c|c|c|c|c|c|c|c|c|c|}
\hline & 2000 & 2001 & 2002 & 2003 & 2004 & 2005 & 2006 & 2007 & 2008 & 2009 & 2010 & 2011 & 2012 & 2013 \\
\hline Agricultura y ciencias biológicas & 62 & 65 & 63 & 102 & 98 & 92 & 129 & 157 & 196 & 193 & 184 & 245 & 233 & 282 \\
\hline Tierra y ciencias planetarias & 11 & 17 & 28 & 34 & 55 & 51 & 73 & 44 & 76 & 89 & 81 & 72 & 94 & 82 \\
\hline Ingeniería & 5 & 5 & 3 & 8 & 13 & 4 & 5 & 12 & 7 & 6 & 28 & 16 & 19 & 11 \\
\hline Ciencias ambientales & 13 & 21 & 27 & 36 & 35 & 31 & 52 & 48 & 48 & 63 & 71 & 100 & 73 & 96 \\
\hline Medicina & 98 & 72 & 87 & 153 & 164 & 172 & 240 & 234 & 203 & 368 & 404 & 452 & 478 & 511 \\
\hline Física y astronomía & 24 & 26 & 19 & 39 & 36 & 32 & 41 & 62 & 33 & 52 & 67 & 53 & 62 & 80 \\
\hline Ciencias sociales & 25 & 29 & 18 & 25 & 28 & 27 & 48 & 47 & 47 & 57 & 80 & 98 & 106 & 99 \\
\hline Inmunología y microbiología & 26 & 20 & 26 & 48 & 35 & 51 & 69 & 59 & 68 & 71 & 64 & 74 & 107 & 90 \\
\hline Ciencias de computación & 2 & 10 & 2 & 7 & 12 & 15 & 19 & 28 & 36 & 30 & 36 & 53 & 47 & 28 \\
\hline Artes y humanidades & 11 & 10 & 5 & 12 & 11 & 19 & 16 & 18 & 21 & 22 & 34 & 40 & 45 & 40 \\
\hline Neurociencias & 1 & 1 & 2 & 2 & 3 & 7 & 10 & 11 & 10 & 8 & 5 & 10 & 8 & 19 \\
\hline Negocios, admin. y contabilidad & 3 & 2 & 0 & 5 & 6 & 8 & 4 & 9 & 13 & 16 & 16 & 23 & 18 & 33 \\
\hline Totales & 281 & 278 & 280 & 471 & 496 & 509 & 706 & 729 & 758 & 975 & 1.070 & 1.236 & 1.290 & 1.371 \\
\hline
\end{tabular}

Datos de SJR, 2015

repuntando al final del período analizado. Aunque el crecimiento presenta una evolución irregular, durante los últimos 8 años analizados el crecimiento fue significativamente superior (en torno a 500 artículos de diferencia). Respecto a los países de Latinoamérica, Ecuador se encuentra en el puesto 12 tanto en producción absoluta como en documentos citables, por detrás de Colombia y Perú que ocupan el quinto y décimo lugar respectivamente.

El crecimiento promedio de la producción científica ecuatoriana experimentado en el período analizado en las áreas temáticas relevantes es del 5,86\%. Este crecimiento puede estar motivado por varias causas:

- el aumento de la inversión que experimenta el Sistema de Educación Superior (especialmente en las universidades públicas) y su mejoría en la coordinación de transferencia en la asignación a actividades generadoras de conocimiento, lo que supone un aumento de recursos para la mejora de las estructuras de investigación, y especialmente para el fomento de proyectos con financiación pública a través de la Senescyt (Secretaría Nacional de Ciencia y Tecnología del Gobierno Ecuatoriano). Y, por otro lado, supone también el crecimiento de la financiación proveniente de

Tabla 9. Comparación de citas totales por áreas temáticas del año 2013

\begin{tabular}{|l|r|r|r|}
\hline & Ecuador & Colombia & Perú \\
\hline Agricultura y ciencias biológicas & 996 & 294 & 173 \\
\hline Tierra y ciencias planetarias & 324 & 82 & 69 \\
\hline Ingeniería & 84 & 196 & 21 \\
\hline Ciencias ambientales & 527 & 210 & 79 \\
\hline Medicina & 805 & 610 & 290 \\
\hline Física y astronomía & 420 & 958 & 138 \\
\hline Ciencias sociales & 106 & 86 & 10 \\
\hline Inmunología y microbiología & 237 & 123 & 55 \\
\hline Ciencias de computación & 73 & 99 & 3 \\
\hline Artes y humanidades & 10 & 16 & 10 \\
\hline Neurociencias & 43 & 65 & 13 \\
\hline Negocios, admin. y contabilidad & 20 & 17 & 4 \\
\hline
\end{tabular}

Datos de SJR, 2015 los programas de investigación que promueve el gobierno nacional a través de sus institutos de investigación.

- El aumento de la colaboración científica entre universidades ecuatorianas y extranjeras a través del Programa Prometeo, que vincula científicos de alta trayectoria de otros países con instituciones de Ecuador, para que potencien y desarrollen la investigación y que sea sustentable en el tiempo. En algunos casos estos investigadores tras finalizar su colaboración temporal se establecen definitivamente en el país, lo que garantiza la viabilidad posterior de los programas y proyectos colaborativos con sus universidades de origen. Esto hace pensar que las redes de colaboración científica creadas permitirán que Ecuador presente a corto plazo un crecimiento en su producción científica, junto a una mejora de la gestión académica y de las políticas de generación de conocimiento. Este hecho debería repercutir positivamente no sólo en el aumento de su producción sino también en su visibilidad.

- La exigencia del sistema ecuatoriano de educación superior aplicado, mediante incentivos y promociones, al personal docente e investigador de las universidades e institutos de investigación. En ese sistema, el principal criterio para la obtención de reconocimiento y promoción son las publicaciones científicas de calidad, siendo las mejor valoradas las indexadas en bases de datos internacionales y de impacto (presencia en la WoS y en Scopus).

\section{Especialización}

El análisis de las áreas temáticas permite conocer las disciplinas más destacadas y establecer la caracterización científica de Ecuador. La mitad de la producción se adscribe a agricultura y medicina, mostrándose como las principales áreas del desarrollo científico nacional y situando a Ecuador en el tercer eje del triángulo de la ciencia. Sucede un hecho similar en Colombia y Perú, aspecto determinado por las semejanzas geográficas ambientales y culturales de los 3 países andinos.

Mantienen una posición predominante agricultura y ciencias biológicas, medicina y ciencias ambientales en los tres 
países analizados. En el caso de Ecuador, aunque las universidades más representativas son de carácter politécnico [Escuela Superior Politécnica del Litoral (Espol) y Escuela Politécnica $\mathrm{Na}$ cional (EPN)], no son las más productivas en publicaciones científicas. Son otras áreas menos técnicas las que cuentan con mayor representatividad y visibilidad. Esto, que en principio resulta llamativo, puede explicarse por el trabajo de investigación que llevan

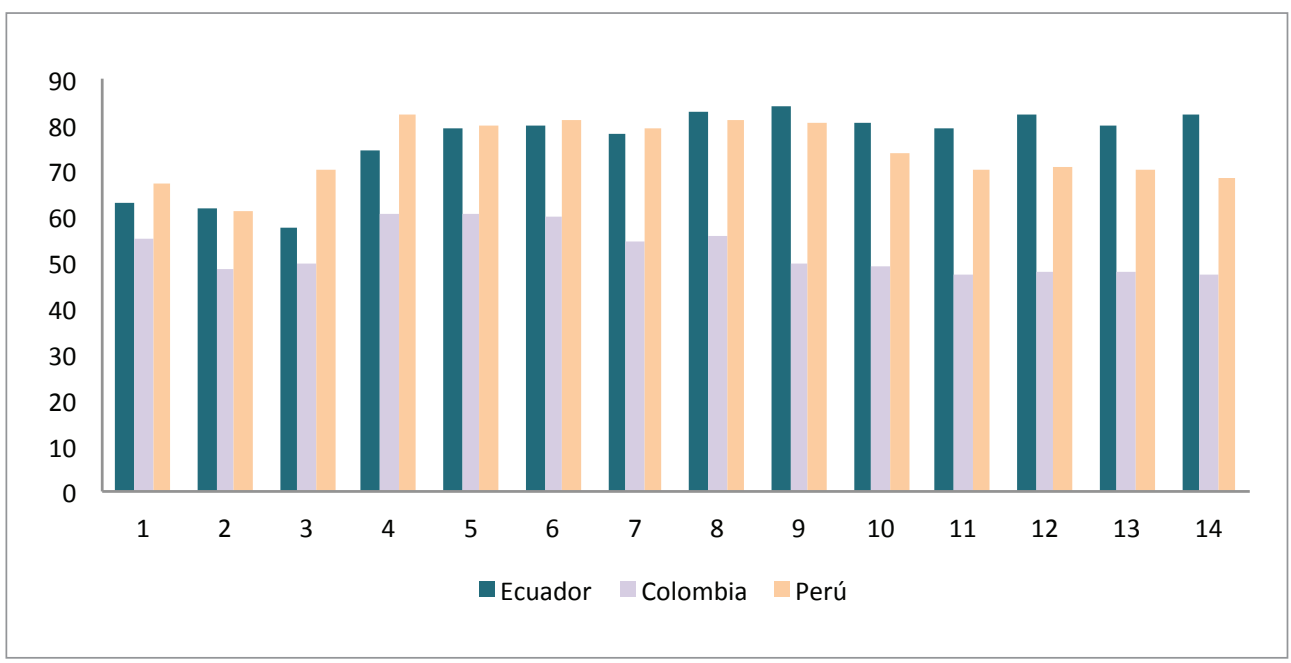

Figura 4. Comparación de citas totales por áreas temáticas del año 2013. Datos de SJR, 2015

a cabo centros como Instituto Nacional de Investigaciones Agropecuarias (Iniap), Centro de Investigaciones de la Caña de Azúcar (Cincae), Centro Nacional de Acuicultura e Investigaciones Marinas (Cenain), Instituto Nacional de Investigación y Salud Pública (Inspi) o Instituto de Neurociencias, entre otros. Su trabajo ayuda a fortalecer el arraigo de áreas biomédicas y de agricultura.

Las redes de colaboración científica permitirán que Ecuador presente un crecimiento en su producción científica, junto a una mejora de la gestión académica y de las políticas de generación de conocimiento

\section{Impacto y la visibilidad}

El uso de la citación para describir la actividad científica debe contemplar la ambigüedad asociada a este indicador y considerarlo en la interpretación de la realidad que describe (Codina-Canet; Perianes-Rodríguez, 2012). Hay que considerar que la frecuencia con la que se cita un artículo depende tanto de la importancia del área científica como de la calidad del trabajo (Sancho, 1990; Campanario; Cabos; Hidalgo, 1998). En este sentido, trabajos de áreas muy pequeñas tendrían pocas posibilidades de tener una alta visibilidad. Estos detalles son importantes para poder realizar una comparación objetiva de los datos de citación de las distintas áreas temáticas.

Los resultados observados no se corresponden con lo esperado, teniendo en cuenta el perfil temático que Ecuador ha presentado durante varias décadas. Según esa trayectoria temática, se esperaría que el área de ciencias sociales fuese la que repuntara. Esta situación se justificaría por la idiosincrasia institucional de no estimular la creación de ciencia y su difusión, sumado a los hábitos de concurrir y celebrar congresos y reuniones científicas sin un nivel de impacto destacable. El retroceso de la visibilidad se corresponde con un aumento de trabajos publicados en revistas no indexadas en bases de datos internacionales, además de trabajos que se publican en forma de comunicaciones y congresos que no tienen el nivel de rigurosidad e impacto requerido. A su vez el $98 \%$ de los trabajos se publican en español y no en inglés.

La colaboración internacional tiene una relación directa con la calidad de las publicaciones científicas de impacto. Los países andinos en el período analizado han presentado altos porcentajes de colaboración. El mayor promedio de colaboración internacional es el de Ecuador con 75,98\%, seguido por Perú con $73,91 \%$ y Colombia con $52,37 \%$. El caso de Ecuador es particular, ya que este alto porcentaje en gran parte es provocado por la inversión del gobierno en el antes citado Proyecto Prometeo, que hasta la fecha ha vinculado 1.800 científicos de los mejores centros de investigación del mundo a las universidades públicas y mantiene una proyección de vincular en 2017 a 5.000 científicos.

Para concluir, se enuncian algunas buenas prácticas para mejorar la productividad y visibilidad científica de los países latinoamericanos:

- potenciar la colaboración científica a través de convenios con universidades con alto prestigio académico e investigativo en las regiones de mayor crecimiento en la generación de conocimiento;

- tener un plan de marketing científico para posicionar la universidad en el mapa académico;

- ejecutar prácticas de socialización de los productos del mercado más competitivos (selección de revistas) que sirvan como medio de difusión de resultados;

- fomentar la creación de repositorios institucionales con el objetivo de maximizar la visibilidad científica;

- instaurar un plan de mejora continua de posicionamiento de la web institucional;

- incorporar nuevos investigadores activos internacionalmente;

- postular la indexación de revistas propias dentro de las bases de datos internacionales más prestigiosas para mejorar el posicionamiento posterior;

- implementar un plan de incentivos a los investigadores con el fin de que la productividad científica tenga el mayor rendimiento. 


\section{Nota}

Este trabajo tiene como eje exclusivamente los resultados obtenidos a partir de las investigaciones científicas publicadas, es decir, sólo analiza aquellos resultados que utilizan las revistas científicas como vehículo de comunicación y que están en el SClmago journal \& country rank $(S J R)$.

\section{Bibliografía}

Bosman, Jeroen; Van-Mourik, Ineke; Rasch, Menno; Sieverts, Eric; Verhoeff, Huib (2006). Scopus reviewed and compared. Utrech: University Library.

http://goo.gl/ZoflOh

Brunner, José-Joaquín; Ferrada-Hurtado, Rocío (eds.) (2011). Educación superior en Iberoamérica. Informe 2011. Providencia, Santiago: Centro Interuniversitario de Desarrollo (Cinda).

http://www.cinda.cl/informes-educacion-superior-eniberoamerica

Bruque-Gámez, Sebastián (2013). La producción científica del Ecuador en el contexto latinoamericano. Un análisis bibliométrico comparativo.

http://goo.gl/qq8AMb

Campanario, Juan-Miguel; Cabos, William; Hidalgo, Miguel-Ángel (1998). "El impacto de la producción científica de la Universidad Alcalá de Henares". Revista española de documentación científica, v. 21, n. 4, pp. 402-405.

http://redc.revistas.csic.es/index.php/redc/article/ viewArticle/361

Codina-Canet, María-Adelina; Olmeda-Gómez, Carlos; Perianes-Rodríguez, Antonio (2013). "Análisis de la producción científica y de la especialización temática de la Universidad Politécnica de Valencia. Scopus (2003-2010)". Revista española de documentación científica, v. 36, n.3, e019.

http://dx.doi.org/10.3989/redc.2013.3.942

Codina-Canet, María-Adelina; Perianes-Rodriguez, Antonio (2012). "Análisis de la colaboración científica de la Universidad Politécnica de Valencia (Scopus, 2003-2008)". Métodos de información, II, v. 3, n. 4, pp. 87-105.

http://dx.doi.org/10.5557/IIMEI2-N2-087105

Concytec (Consejo Nacional de Ciencia, Tecnología e Innovación Tecnológica) (2014). Informe No. 1 Principales indicadores bibliométricos de la actividad científica peruana 2006-2011.

http://goo.gl/tq1xsl

Conicyt (Comisión Nacional de Investigación Científica y Tecnológica) (2014). Principales indicadores cienciométricos de la actividad científica chilena 2012. Informe 2014: una mirada a 10 años. http://www.conicyt.cl/informacioncientifica/files/2014/08/ Informe-de-Chile-2012.pdf

Correa, Rafael (2009). Ecuador: de Banana Republic a la No República. Debate. ISBN: 9789588613376

Herrero-Solana, Víctor; Vargas-Quesada, Benjamín (2010). "Especialización temática de la producción científica. En: Sanz-Menéndez, Luis; Cruz-Castro, Laura. Análisis sobre ciencia e innovación en España. Madrid: Instituto de Políticas y Bienes Públicos (IPP) del CSIC, pp. 258-275.

Levy, Daniel (2011). "Las múltiples formas de educación superior privada: Un análisis global”. En: Brunner, JoséJoaquín; Peña, Carlos (eds.). El conflicto de las universidades: entre lo público y lo privado. Santiago de Chile: Ediciones Universidad Diego Portales. ISBN: 978956314 1313

Ministerio de Educación de Colombia (2013). Estadísticas de Educación Superior al 31 de diciembre del 2013

http://www.mineducacion.gov.co/sistemasdeinformacion/1735/ w3-article-346086.html

Molina-Molina, Silvia; De-Moya-Anegón, Félix (2013). “Política nacional y visibilidad internacional. El caso colombiano". El profesional de la información, v. 22, n. 6, pp. 529-535 http://www.elprofesionaldelainformacion.com/contenidos/2013/ noviembre/05.pdf

http://dx.doi.org/10.3145/epi.2013.nov.05

OECD (2012). Education at a glance 2012. Paris: OECD. ISBN: 9789264179295

http://www.oecd.org/edu/EAG\%202012_e-book_EN_200912. $p d f$

Ricyt (2015). Indicadores bibliométricos. Buenos Aires, Red de Indicadores de Ciencias y Tecnologías Iberoamericanas e Interamericanas.

http://www.ricyt.org/indicadores

Sancho, Rosa (1990). "Indicadores bibliométricos utilizados en la evaluación de la ciencia y tecnología. Revisión bibliográfica". Revista española de documentación científica, v. 13, n. 3-4, pp. 842-865.

http://digital.csic.es/handle/10261/23694

Secretaría Nacional de Planificación y Desarrollo (2015). Ocho años de la revolución ciudadana del conocimiento. Quito. http://goo.gl/HBjmHi

Senescyt (2013). Recursos presupuesto general del estado para instituciones de Educación Superior 2013. Secretaría Nacional de Educación Superior Ciencia, Tecnología e Innovación. http://goo.gl/SSV9OV

SJR (2014). SCImago journal \& country rank. http://www.scimagojr.com/countryrank.php

SJR (2015). SCImago journal \& country rank. http://www.scimagojr.com 